\title{
Real-time detection of individual atoms falling through a high-finesse optical cavity
}

\author{
H. Mabuchi, Q. A. Turchette, M. S. Chapman, and H. J. Kimble \\ Norman Bridge Laboratory of Physics 12-33, California Institute of Technology, Pasadena, California 91125
}

Received April 22, 1996

\begin{abstract}
The enhanced coupling between atoms and photons inside a high-finesse optical cavity provides a novel basis for optical measurements that continuously monitor atomic degrees of freedom. We describe an experiment in which cavity quantum-electrodynamic effects are utilized for real-time detection of individual atoms falling through an optical cavity after being dropped from a magneto-optical trap. Our technique permits experiments that are triggered by the presence of a single optimally coupled atom within the cavity mode volume. (C) 1996 Optical Society of America
\end{abstract}

Although the earliest experiments in cavity quantum electrodynamics (QED) investigated alterations of atomic radiative processes in the presence of external boundaries, ${ }^{1}$ subsequent studies have placed equal emphasis on the complementary alterations of a cavity's electromagnetic response caused by near-resonant interaction with atoms. From its beginnings in the optical bistability literature, ${ }^{2}$ the latter program of research has advanced to a modern era of cavity QED systems in which significant modifications can be observed with a mean intracavity atom number much less than $1 .^{3} \quad$ Recent experiments ${ }^{4-7}$ have begun to explore the strong-coupling regime in which the single-photon Rabi frequency $2 g_{0}$ for a single intracavity atom dominates the cavity field decay rate $\kappa$, the atomic dipole decay rate $\gamma_{\perp}$, and the inverse atomic transit time $T^{-1}$. Current efforts focus on extending these initial demonstrations of strong coupling into realizations of quantum state synthesis, ${ }^{8}$ quantum logic, ${ }^{9}$ and quantum nondemolition measurement. ${ }^{10}$

Despite this significant progress, in no cavity QED experiment to date have single-atom effects been observed without integration over the transits of many successive atoms through a cavity. By contrast, we report in this Letter an experiment that utilizes strong coupling to achieve real-time detection of individual atoms, which we drop from a magneto-optic trap $^{11}$ (MOT) into a high-finesse optical resonator. In addition to representing an important technical advance within the context of cavity QED, our experiment demonstrates a high-bandwidth, all-optical technique for continuously monitoring the trajectories of moving atoms. We easily resolve the motion of a single atom over distances of $100 \mu \mathrm{m}$ in $100 \mu \mathrm{s}$, with evidence for submicrometer resolution on $10-\mu$ s time scales in optimal cases. Theoretical treatments of similar cavity-QED schemes ${ }^{12,13}$ have shown that it should ultimately be possible to reach and perhaps exceed the standard quantum limit for atomic position measurement. ${ }^{14}$ Within the broader context of singleatom detection by absorption, note that in our research single atoms are detected in times $\sim 10^{5}$ shorter than in previous demonstrations. ${ }^{15}$

To perform single-atom detection we monitor the transmission of a resonant cavity probe laser after dropping atoms from a MOT formed over the gap between the cavity mirrors (see Fig. 1). Before the arrival of the freely falling atoms the cavity transmits a constant mean power $P_{0}$, which is determined with a balanced heterodyne detector. Small variations in the detected power arise from heterodyne shot noise and from technical imperfections in the cavity locking. After a mean time delay corresponding to that required for atoms to fall from MOT to the cavity axis the transmitted power exhibits distinct dips, which we attribute to intracavity absorption by single atoms falling through the resonator mode volume. No such events are observed in data taken with the MOTloading atomic beam blocked or with the polarization of one trapping beam changed to prevent formation of the MOT. In Fig. 2 we display an example of the time-varying heterodyne signal with an inset that shows a histogram of atom arrival times for a typical run of 450 trap-drop cycles (average 2.5 events/drop). The variance $( \pm 7 \mathrm{~ms})$ of the arrival-time distribution is as expected for an atomic sample cooled to the Doppler limit.

The physical origin of the atom-transit signals can be understood with reference to either the JaynesCummings Hamiltonian ${ }^{16}$ or the optical bistability state equation. ${ }^{2,13}$ For the case of equal atomic, cavity, and probe frequencies $\left(\omega_{A}=\omega_{C}=\omega_{L}\right)$, both models predict that atomic absorption of intracavity photons should decrease the cavity transmission to a steady-

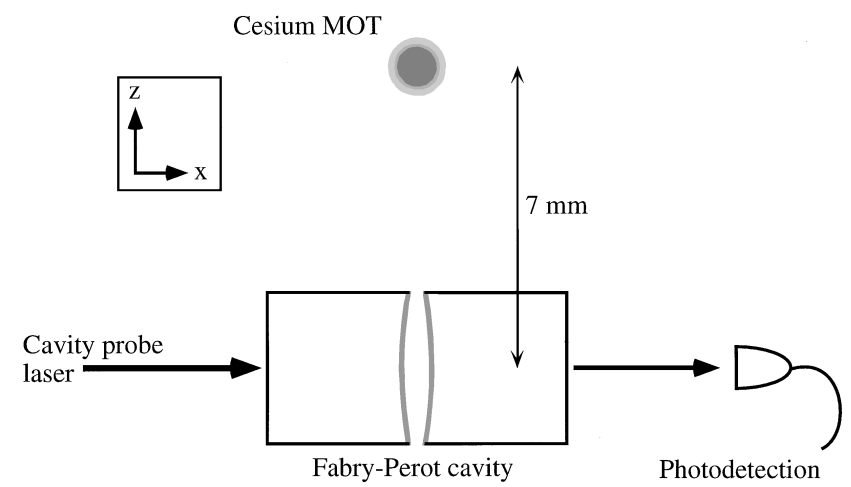

Fig. 1. Schematic diagram of the experiment. The cesium MOT lies $7 \mathrm{~mm}$ above the cavity axis, the mirror substrate diameter is $3 \mathrm{~mm}$, and the cavity length is $108 \mu \mathrm{m}$. 


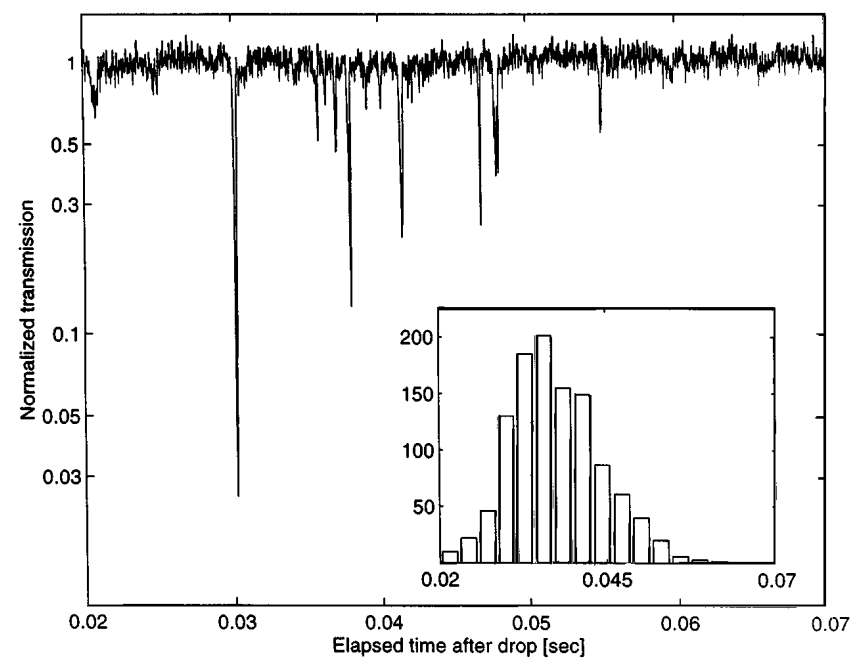

Fig. 2. Time-varying cavity transmissions after a cesium MOT is dropped, normalized to probe power $P_{0}$ transmitted through the empty cavity. For these data $P_{0}$ corresponds to $\approx 2$ photons in the empty cavity, and $\omega_{A}=\omega_{C}=\omega_{L}$ (see text). Inset: histogram of atom arrival times compiled from 450 consecutive trap-drop cycles.

state value that depends on the atom-cavity coupling $g$. As $g$ depends on the atom's position within the cavity, the time-varying transmission carries realtime information on the trajectory $g[\mathbf{r}(t)]$ of an atom traversing the cavity mode. For our Fabry-Perot cavity, $g(\mathbf{r}) \equiv g_{0} \psi(x, y, z) \simeq g_{0} \cos (2 \pi x / \lambda) \exp \left[-\left(y^{2}+\right.\right.$ $\left.\left.z^{2}\right) / w_{0}^{2}\right]$, where $w_{0} \simeq 45 \mu \mathrm{m}$ and $\lambda=852.359 \mathrm{~nm}$ for the cesium $\left(6 S_{1 / 2}, F=4\right) \rightarrow\left(6 P_{3 / 2}, F^{\prime}=5\right)$ transition (for which $\gamma_{\perp} / 2 \pi=2.5 \mathrm{MHz}$ ).

To detect single atoms within their transit time $T$ we require that $\mathcal{N} \equiv T \kappa m_{0}|Y-X| \gg 1$, where for fixed incident probe power $m_{0} Y$ represents the photon number that builds up in an empty cavity and $m_{0} X$ represents the reduced photon number with one optimally coupled atom. In our cavity $m_{0} \equiv 4 \gamma_{\perp}^{2} / 3 g_{0}{ }^{2} \approx 0.07$. For good photon-counting statistics the transmitted photon-flux difference $\kappa m_{0}|Y-X|$ must be large, but atomic saturation leads to greatly reduced contrast $(Y-X) / Y$ for $Y \gg C^{2}$. There thus exists an optimal value for the incident probe power. We have numerically investigated steady-state solutions of the master equation for our system ${ }^{17}$ and find that, with our experimental parameters $\left(g_{0}, \kappa, \gamma_{\perp}, T^{-1}\right) / 2 \pi=$ $(11,3.5,2.5,0.001) \mathrm{MHz}$ (see below), the condition on $\mathcal{N}$ should be well satisfied for $0.1 \leq m_{0} Y \leq 10$. For the data of Fig. 2, $m_{0} Y \approx 2$.

The Fabry-Perot resonator used in our experiment consists of two superpolished spherical mirrors (radius of curvature $1 \mathrm{~m}$ ) with dielectric high-reflectivity coatings (15 parts in $10^{6}$ residual transmission and loss). After being coated, the mirrors were machined down to 3 -mm diameter, allowing for a low-profile cavity that can be positioned well within $1 \mathrm{~cm}$ of the trapped cesium atoms. The cylindrical mirror subrates sit in a machined groove atop an aluminum mount that is passively isolated from mechanical vibrations. We measured the cavity length to be $108 \mu \mathrm{m}$ and the linewidth of the $\mathrm{TEM}_{00}$ mode to be $\kappa / 2 \pi=3.5 \pm$ $1 \mathrm{MHz}$ (HWHM), consistent with the nominal cavity finesse $\mathcal{F}=2 \times 10^{5}$. The mirrors used in the current experiment exhibit significant birefringence (possibly because of machining stresses), which leads to an observed polarization-mode splitting of $\sim 7 \mathrm{MHz}$ for the $\mathrm{TEM}_{00}$ spatial mode. The Gaussian waist of the cavity mode is computed from geometry to be $45 \mu \mathrm{m}$, which when combined with atomic parameters for the cesium $\left(6 S_{1 / 2}, F=4, m_{F}=4\right) \rightarrow\left(6 P_{3 / 2}, F^{\prime}=5, m_{F}{ }^{\prime}=5\right)$ transition yields $g_{0} / 2 \pi \simeq 11 \mathrm{MHz}$. Note that reduced values of the coupling are obtained for other Zeeman transitions within the $F=4 \rightarrow F^{\prime}=5$ manifold.

Our MOT employs a six-beam configuration and loads directly a thermal atomic beam. We orient the anti-Helmholtz coils for the trap so that their symmetry axis is parallel to that of the optical cavity. This leads to a MOT laser-beam geometry with one beam axis running parallel to and just above the cavity and two beam axes in the plane of the mirror surfaces. We estimate an average number of atoms $\approx 1 \times 10^{5}$ from CCD images taken during the experiment. The data acquisition cycle begins with a trap-loading period of $\sim 1 \mathrm{~s}$, at the end of which the anti-Helmholtz field is ramped to zero (within $3 \mathrm{~ms}$ ) and the trapping beams are turned off with an acousto-optic modulator and a mechanical shutter. The repumping beam is left on so that the falling atoms are pumped into the $F=4$ ground hyperfine level. The resonant probe beam used to monitor the cavity transmission is generated by $\mathrm{rf}$ synthesis techniques ${ }^{18}$ from a stabilized Ti:sapphire laser, and we measure the transmitted power by using balanced heterodyne detectors with an overall efficiency of $\approx 0.2$ and $100-\mathrm{kHz}$ bandwidth. The resulting signal is digitized at $500 \mathrm{kHz}$ by a PCbased acquisition system. An error signal is derived directly from the photocurrent to stabilize the cavity length and fix the $\mathrm{TEM}_{00}$ resonance frequency to coincide with atomic resonance. Each trap-drop cycle produces a time series like the one shown in Fig. 2.

In Fig. 3 we show close-up views of six individual atom-transit signals. We associate the broad envelope exhibited by all our transit signals with the Gaussian variation of $g(\mathbf{r})$ in the vertical dimension and note that many signals display additional, highly structured variations within this overall envelope. For example, the signals in Figs. 3(b), 3(c), and 3(e) contain very regular, large-amplitude oscillations, which we tentatively attribute to atomic motion through the cavity's standing wave. Based on the experimental geometry indicated in Fig. 1 and on a MOT temperature of $\sim 100 \mu \mathrm{K}$, we expect that many of the atoms entering the cavity will have initial velocities of $\sim 1 \mathrm{~cm} / \mathrm{s}$ along the cavity axis. If recoil-induced heating by the intracavity field does not significantly increase a given atom's axial velocity, the sinusoidal variation of $g(\mathbf{r})$ should lead to an oscillation of the transmitted probe power on $\sim 10-\mu \mathrm{s}$ time scales. Evidence for such oscillations can be found throughout the data that we have taken. Although some of the structure seen in Fig. 3 could be attributed to shot noise or to technical noise, a comparison between pairs of signals such as those in Figs. 3(a) and 3(b) reveals that the variations observed in an oscillating signal can greatly exceed the noise amplitude that would be predicted from 


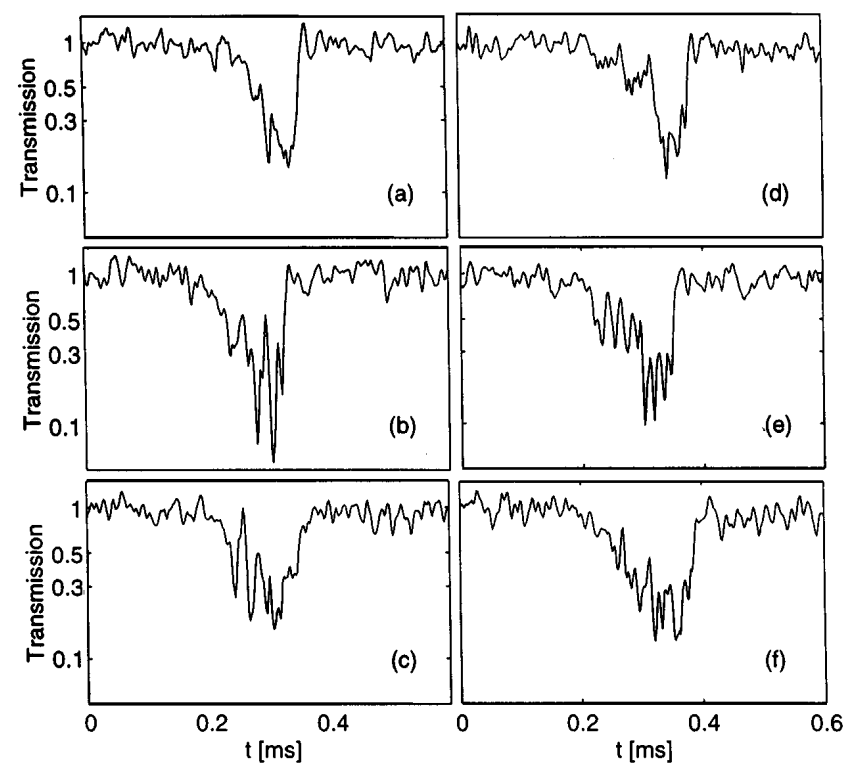

Fig. 3. Six examples of transit signals associated with the passage of individual atoms through the optical cavity. The data are normalized and taken with the same parameters as for Fig. 2, except that $P_{0}$ corresponds to three intracavity photons.

an examination of signals with a more smoothly varying envelope. Beyond spatial variations in $g(\mathbf{r})$ we expect that the complexity of possible transitions within the $F=4 \rightarrow F^{\prime}=5$ Zeeman manifold will contribute additional signal structure. A majority of the transit signals are quite asymmetric, often with a steep and structureless trailing edge as in Figs. 3(a) and 3(b). A simple calculation supports the association of such edges with optical pumping to the uncoupled $F=3$ hyperfine ground state by means of off-resonant excitation of $F=4 \rightarrow F^{\prime}=4$ transitions.

The magnitudes of the signals that we observe are qualitatively consistent with our numerical solutions of the master equation. Unfortunately, we have been unable to make quantitative comparisons with theory because the aforementioned birefringence of our cavity prevents us from realizing the two-state atomic dynamics assumed by standard theoretical models.

In summary, we have combined laser-cooling techniques with cavity QED to achieve real-time detection of individual atoms as they fall through a high-finesse optical cavity. Future cavity-QED experiments can use this method to circumvent fluctuations of intracavity atom number by being triggered by isolated events. Modest improvements in detection efficiency should permit continuous measurement of individual atomic trajectories with sensitivity approaching the standard quantum limit, opening the possibility for quantum feedback. ${ }^{19}$ In contrast to single-atom maser- and laser-type experiments, ${ }^{4,6,7}$ our configuration achieves atom dwell times that greatly exceed all system time scales (e.g., $g T \approx \pi$ in Refs. 4, 6, and 7 compared with $g T \approx 10^{4}$ here). Hence the atom-cavity system can be cycled through many different equilibrium states during a single atomic transit. Finally, we note that showing an atom just before it enters the cavity could raise the ratio $\hbar g_{0} / E_{k}$ of the single-photon coupling en- ergy to the atom's total kinetic energy from its current value of $\approx 0.2$ to values of $\geq 1$. This would permit the investigation of photon-covalent mechanical binding of atom and cavity. ${ }^{20}$

We gratefully acknowledge our ongoing collaboration with A. S. Parkins, who encouraged us to initiate the research described in this Letter. We thank W. Lange and C. Hood for valuable technical assistance as well as R. Lalezari and co-workers at Research Electrooptics for mirror fabrication. H. Mabuchi holds a National Defense Science and Engineering graduate fellowship. M. S. Chapman acknowledges support from a Millikan Prize fellowship. This research is supported by the U.S. Office of Naval Research and the National Science Foundation.

\section{References}

1. K. H. Drexhage, in Progress in Optics, E. Wolf, ed. (North-Holland, Amsterdam1974), Vol. 22, p. 163; E. A. Hinds, in Advances in Atomic, Molecular, and Optical Physics 28, D. Bates and B. Bederson, eds. (Academic, New York, 1990), p. 237.

2. L. A. Lugiato, in Progress in Optics, E. Wolf, ed. (NorthHolland, Amsterdam, 1984), Vol. 21, p. 71.

3. See Cavity Quantum Electrodynamics, P. R. Berman, ed. (Academic, San Diego, 1994), p. 203.

4. G. Rempe, F. Schmidt-Kaler, and H. Walther, Phys. Rev. Lett. 64, 2783 (1990); see also Ref. 3.

5. R. J. Thompson, G. Rempe, and H. J. Kimble, Phys. Rev. Lett. 67, 1132 (1992).

6. K. An, J. J. Childs, R. R. Dasari, and M. S. Feld, Phys. Rev. Lett. 73, 3375 (1994).

7. M. Brune, F. Schmidtkaler, A. Maali, J. Dreyer, E. Hagley, J. M. Raimond, and S. Haroche, Phys. Rev. Lett. 76, 1800 (1996); see also Ref. 3.

8. A. S. Parkins, P. Marte, P. Zoller, and H. J. Kimble, Phys. Rev. Lett. 71, 3095 (1993); C. K. Law and J. H. Eberly, Phys. Rev. Lett. 76, 1055 (1996).

9. Q. A. Turchette, C. J. Hood, W. Lange, H. Mabuchi, and H. J. Kimble, Phys. Rev. Lett. 75, 4710 (1995).

10. M. Brune, P. Nussenzveig, F. Schmidtkaler, F. Bernadot, A. Maali, J. M. Raimond, and S. Haroche, Phys. Rev. Lett. 72, 3339 (1994).

11. E. L. Raab, M. Prentiss, A. Cable, S. Chu, and D. E. Pritchard, Phys. Rev. Lett. 59, 2631 (1987).

12. P. Storey, M. Collett, and D. F. Walls, Phys. Rev. Lett. 68, 472 (1992); M. A. M. Marte and P. Zoller, Appl. Phys. B 54, 477 (1992).

13. G. Rempe, Appl. Phys. B 60, 233 (1995).

14. M. Ozawa, Phys. Rev. Lett. 60, 385 (1988).

15. D. J. Wineland, W. M. Itano, and J. C. Bergquist, Opt. Lett. 12, 389 (1987); W. E. Moerner and L. Kador, Phys. Rev. Lett. 62, 2535 (1989).

16. E. T. Jaynes and F. W. Cummings, Proc. IEEE 51, 89 (1963); P. Alsing and H. J. Carmichael, Quantum Opt. 3, 13 (1991).

17. H. J. Carmichael, An Open Systems Approach to Quantum Optics (Springer-Verlag, Berlin, 1993).

18. Q. A. Turchette, R. J. Thompson, and H. J. Kimble, Appl. Phys. B 60, S1 (1995).

19. H. M. Wiseman and G. J. Milburn, Phys. Rev. Lett. 70, 548 (1993).

20. S. Haroche, M. Brune, and J. M. Raimond, Europhys. Lett. 14, 19 (1991); B. G. Englert, J. Schwinger, A. O. Barut, and M. O. Scully, Europhys. Lett. 14, 25 (1991). 